\title{
Contingency Ranking for Voltage Collapse via Sensitivities from a Single Nose Curve
}

\author{
Scott Greene \\ Ian Dobson \\ Member \\ Fernando L. Alvarado \\ Student Member \\ Electrical and Computen \\ University of Wisconsin, Madison WI 53706 USA
}

\begin{abstract}
The change in the loading margin to voltage collapse when line outages occur is estimated. First a nose curve is computed by continuation to obtain a nominal loading margin. Then linear and quadratic sensitivities of the loading margin to each contingency are computed and used to estimate the resulting change in the loading margin. The method is tested on a critical area of a 1390 bus system and all the line outages of the IEEE 118 bus system. The results show the effective ranking of contingencies and the very fast computation of the linear estimates.
\end{abstract}

Keywords: Power system security, contingency analysis, sensitivity, voltage stability, bifurcation, power system dynamic stability

\section{Introduction}

Contingencies such as unexpected line outages often contribute to voltage collapse blackouts [1]. These contingencies generally reduce or even eliminate the voltage stability margin. To maintain security against voltage collapse, it is desirable to estimate the effect of contingencies on the voltage stability margin. Action can then be taken to increase the margin so that likely contingencies do not cause blackout.

Suppose that the power system is operating stably at a certain loading level referred to as the "base case loading". By means of a short term load forecast or otherwise, assume a particular pattern of load increase. The amount of additional load in this direction that would cause a voltage collapse is called the loading margin to voltage collapse. The curve marked "nominal" in Figure 1 shows a specific bus voltage as a function of total system loading. The nose of the curve is associated with voltage collapse $[2,3]$ and the nominal loading margin is the megawatt distance between the base case loading and the loading at the nose. The loading margin is computed by tracing the nose curve and finding the nose.

Suppose that a contingency such as loss of a line occurs at the base case loading. Assuming that the system restabilizes after the transient, the voltage as a function

PE-707-PWRS-2-06-1997 A paper recommended and approved by the IEEE Power System Dynamic Performace Committee of the IEEE Power Engineering Society for publication in the IEEE Transactions on Power Systems. Manuscript submitted November 8, 1996; made available for printing June 11, 1997. of loading changes to the curve marked "contingency" in Figure 1. Since the contingency causes the nose to move to a lower loading, the loading margin is reduced. If the loading margin is thought of as a smooth function of the line admittance, then the sensitivity of the loading margin with respect to changes in the line admittance can be calculated at the nominal nose. This sensitivity can then be used to estimate the change in the loading margin due to the changes in admittance caused by the line outage. This approach avoids retracing the nose curve to compute the loading margin with the line removed.

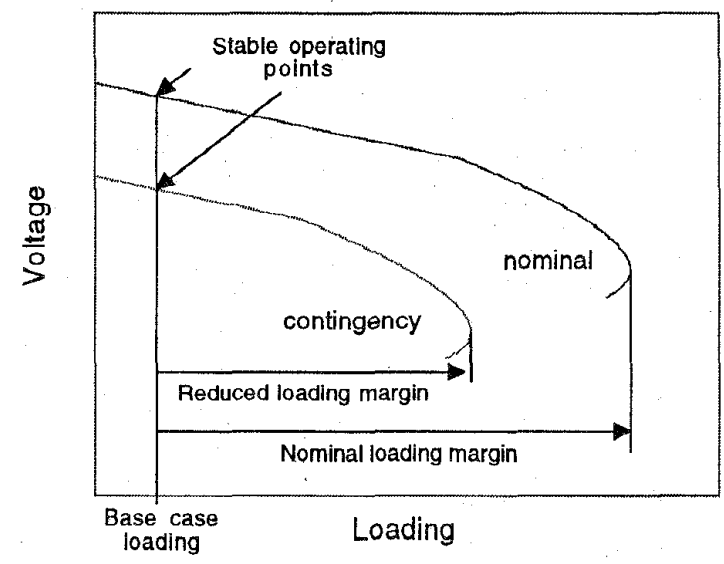

Figure 1: Nominal and contingency nose curves

The main idea of the paper is to compute a single nose curve and associated sensitivities and to use these to quickly estimate the change in the loading margin to voltage collapse for any line outage. This approach uses the general loading margin sensitivity formulas derived in [4].

The computations are summarized:

1 A pattern of load increase, generator dispatch policy, and area interchange schedule are forecast or assumed.

2 A continuation method such as $[5,6,7,8,9]$ is used to trace the nose curve and hence determine the nominal loading margin.

3 Quantities needed for the sensitivity formulas are evaluated at the nose point. Then, for each contingency, the change in loading margin is estimated by evaluating the sensitivity formulas presented in section 5 .

The accuracy of the estimate can be improved by using quadratic sensitivities. Inaccuracies can occur when 
contingencies shift the voltage collapse area or drastically alter the set of generators which are VAR limited at the nose. These inaccuracies are quantified in the results for the 118 bus test system.

This paper only addresses security with respect to voltage collapse; security concerns such as under voltages, thermal overloads, oscillations and transient stability are not addressed.

\section{Frevious Work}

The overall approach of our paper is similar to and inspired by Wu and Fischl [10]. Wu and Fischl [10] use quadratic estimates to approximate the effect of contingencies on interarea transfer margins for an 11 bus system. An interarea transfer margin can be viewed as a particular and useful choice of a loading margin and the linear estimate of $[10]$ is then equivalent to the linear estimate of this paper. However, the formula of [10] for the quadratic estimate neglects the implicit dependence of the Jacobian on the operating equilibrium and is different than that used in this paper (see closure of [4]). The idea of treating a discrete parameter such as line admittance as a continuous parameter has also been presented in [11]. Second order sensitivities have been used for power system optimization problems in [12] and [13].

Contingency screening and analysis concerning line flow and voltage limit violations, as well as transient stability, have been an active research area for several decades. A comprehensive bibliography is contained in the recent work [14]. Reference [15] provides an exce]lent synopsis of earlier: work.

The effect of contingencies on long term voltage stability is addressed in [16-24]. References $[19,20,22,24]$ specifically address the effect of contingencies on the margin to voltage collapse. Reference [25] presents a method of estimating the loading margin to voltage collapse that is applicable to contingency analysis.

Flatabø et al. [19] describe a sensitivity based method to estimate the margin to voltage instability, and propose performing contingency selection by computing an index dependent upon the post-contingency sensitivity of voltage to reactive power. Fosso et al. [20] compare the method of [19] to an optimization method and a curve fit method to determine the margin to voltage instability. Ejebe et al. [22], motivated by [20], demonstrate computing three load flows per contingency and fitting a curve to determine the loading margin. Chiang et al. [24] perform a similar curve fit with only 2 load flows per contingency. Zeng et al. [25] explain a similar approach and conclude that good results can be obtained with 5 load flow solutions.

The method presented in this paper differs from $[20,22,24,25]$ in that no curve fitting is used, and that post-contingency loading margins are estimated by sensitivity analysis as deviations from a nominal loading margin. The curve fit methods produce voltage profiles so that contingencies can also be screened for voltage magnitude problems as well as stability margin. However, it is difficult to properly account for changes in reactive power limits by fitting curves to only a few equilibrium solutions. The sensitivity method can take account of reactive power limits when the initial continuation computes the nominal nose. The curve fit methods require several load flows to be computed per contingency. The sensitivity methods require a single continuation to find the nominal nose (10 to 100 loadflow solutions) and then require much less computation per contingency. The linear estimate for each contingency is at least three orders of magnitude faster than one load flow solution. The quadratic estimate for each contingency is about equivalent in computational expense to one load flow solution, and thus is faster than any method requiring multiple loadflow solutions per contingency.

The next section describes the test results and is followed by a discussion; the computations are detailed in section 5 .

\section{Results}

The contingency analysis is tested on two systems. A 1390 bus system is used to assess the computation time and practicality of the method. The 118 bus system tests the effects of encountering generator VAR limits and evaluates all possible line outages on a system vulnerable in multiple areas.

For each system, a base case operating point at which the outages are assumed to occur is identified, a given pattern of load increase is assumed, and a nominal loading margin is obtained by locating the nose point with a continuation method. The system loading and loading margin are measured by the sum of all real load powers. The estimates for each contingency are evaluated at the nominal nose point.

To test the accuracy of the estimates, the actual loading margins are computed for each outage as follows: A post-contingency operating point at the base case loading is obtained by solving several load flows, each for a gradually decreased line admittance until the line is completely outaged. This procedure does not necessarily reflect the settling of the actual system transient, but it is a sensible way to identify a plausible post-contingency operating point [8] (the procedure is needed to avoid convergence to a nearby unstable equilibrium). Then a continuation starting from the postcontingency operating point is used to find the nose as the load is increased in the specified direction. All load flow and continuation computations are performed using the PFLOW package [28]. The estimates are computed using MATLAB and the Sparse Matrix Manipulation System $[29,30]$.

\subsection{0 bus system}

The estimates are tested on all non-radial $500 \mathrm{kV}$ line outages in an area of a 1390 bus system thought to be prone to voltage collapse. The 1390 bus system includes more than 2000 transmission lines and more than 200 transformers. The base case operating point at which the outages are assumed to occur has a total system loading of $94097 \mathrm{MW}$ and reflects a very heavily loaded system. Both real and reactive power increase from 
the base case loading at 5 critical buses. The dispatch distributes slack to 25 generators. For this test, transformer taps are assumed fixed at the base case loading and generator VAR limits encountered above the base case loading are ignored. The total system loading at the nominal point of collapse is $95548 \mathrm{MW}$ and corresponds to a loading margin of $1451 \mathrm{MW}$.

The results ordered by severity are shown in Table 1. The quadratic estimate selects the top 5 and top 12 of the most severe outages. The linear estimate selects 3 of the top 5 and 11 of the top 12 . In general, the magnitude of the error increases with the severity of the outages and the actual loading margins are less than the estimates. The result highlighted with slanted typeface indicates a case for which the quadratic estimate captures a critical outage that the linear estimate misses.

The following approximate timings were obtained on a Hewlett Packard 9000 series 700 workstation:

- 1000 linear estimates require $1 \mathrm{CPU}$ second.

- One quadratic estimate requires $15 \mathrm{CPU}$ seconds.

- One iteration of a loadflow requires 2 CPU seconds.

- One loadflow solution requires 5 to 25 CPU seconds. Thus each linear estimate takes negligible time compared to a loadflow iteration whereas each quadratic estimate takes about the same time as a loadflow solution (several loadflow iterations).

\subsection{All single contingencies of the 118 bus sys- tem}

The contingency analysis is next tested using a particular base case and voltage collapse of the 118 bus IEEE test system $[4,27]$. The base case operating point at which the outages are assumed to occur has a total system loading of $5677 \mathrm{MW}$. Both real and reactive loads at 91 buses increase proportionally from the base case loading. 17 generators participate in the dispatch with the slack distributed so that generators in each area provide additional real power roughly in proportion to their size. There are 9 fixed tap transformers. A continuation method is used to obtain the nominal loading margin and generator VAR limits apply as demand increases. The nominal voltage collapse occurs for a total load of $7443 \mathrm{MW}$ and a loading margin of $1766 \mathrm{MW}$. The area interchange is enforced for the entire continuation and the appropriate area interchange equations are included in the computation of the estimates. This case is intended to provide a challenging test for the sensitivity based formulas since the system is stressed in every area, increasing the possibility that seme outages may cause a voltage collapse in a different area than that of the nominal collapse. Changes in generator VAR limits were computed during both the procedure to find the post-contingency operating point and the subsequent continuation.

Linear and quadratic estimates for the postcontingency loading margins are evaluated for all of the possible line outages (177 outages). Two of the contingencies are so severe that a post-contingency operating point does not exist. These two contingencies result in the outage of a critical generator.
Table 1: Estimated loading margins for all $500 \mathrm{kV}$ line outages in a critical area of the 1390 bus system.

$$
\text { nominal loading margin }=1451 \mathrm{MW}
$$

\begin{tabular}{lll}
\hline Linear & Quadratic & Exact \\
estimate & estimate & \\
MW (rank) & MW (rank) & MW (rank) \\
\hline $1124(3)$ & $791(2)$ & $323(1)$ \\
$1083(2)$ & $864(3)$ & $706(2)$ \\
$1072(1)$ & $870(4)$ & $772(3)$ \\
$1258(11)$ & $1078(5)$ & $866(4)$ \\
$1462(29)$ & $439(1)$ & $902(5)$ \\
$1197(6)$ & $1195(9)$ & $973(6)$ \\
$1197(7)$ & $1195(10)$ & $974(7)$ \\
$1219(9)$ & $1194(8)$ & $1018(8)$ \\
$1219(10)$ & $1195(11)$ & $1020(9)$ \\
$1216(8)$ & $1206(12)$ & $1024(10)$ \\
$1184(4)$ & $1170(6)$ & $1047(11)$ \\
$1185(5)$ & $1172(7)$ & $1051(12)$ \\
$1393(16)$ & $1339(14)$ & $1172(13)$ \\
$1416(19)$ & $1405(20)$ & $1209(14)$ \\
$1348(12)$ & $1338(13)$ & $1306(15)$ \\
$1366(13)$ & $1363(15)$ & $1310(16)$ \\
$1366(14)$ & $1363(16)$ & $1311(17)$ \\
$1398(17)$ & $1370(17)$ & $1330(18)$ \\
$1398(18)$ & $1370(18)$ & $1331(19)$ \\
$1379(15)$ & $1377(19)$ & $1369(20)$ \\
$1442(25)$ & $1439(28)$ & $1382(21)$ \\
$1421(20)$ & $1420(23)$ & $1392(22)$ \\
$1436(21)$ & $1417(22)$ & $1393(23)$ \\
$1437(22)$ & $1407(21)$ & $1394(24)$ \\
$1441(23)$ & $1424(24)$ & $1407(25)$ \\
$1441(24)$ & $1425(25)$ & $1407(26)$ \\
$1445(26)$ & $1432(26)$ & $1408(27)$ \\
$1446(27)$ & $1433(27)$ & $1409(28)$ \\
$1450(28)$ & $1448(29)$ & $1428(29)$
\end{tabular}

For the 175 survivable line outages, the mean postcontingency loading margin is $1738 \mathrm{MW}, 28 \mathrm{MW}$ less than the nominal loading margin of $1766 \mathrm{MW}$. The mean absolute error of the linear estimate is $20 \mathrm{MW}$ and the mean absolute error of the quadratic estimate is $16 \mathrm{MW}$. The median error for both the linear and quadratic estimates is less than $1 \mathrm{MW}$.

In order to determine how assumptions concerning generator VAR limits affect the accuracy of the estimates, actual loading margins were also computed enforcing a fixed set of generator limits, determined by those generators that were at VAR limits at the nominal nose point. (The formulas from [4] assume that a fixed set of equations model the equilibrium, i.e. that those generators limited at the nominal nose and only those generators are limited at the post-contingency nose.) The estimates perform better when compared with the results for the set of generators at VAR limits fixed: The mean absolute errors reduce to $13 \mathrm{MW}$ and $10 \mathrm{MW}$ for the linear and quadratic estimates respectively; while the mean actual change in the margin is $21 \mathrm{MW}$. 
The majority of line outages do not significantly affect the loading margin and these are adequately screened by the estimates. Of the 126 outages causing less than a $10 \mathrm{MW}$ change in the loading margin, the mean absolute errors of both the linear and quadratic estimates are less than $1 \mathrm{MW}$. Only 6 of the 126 outages have an error greater than $3 \mathrm{MW}$ and the maximum error is $10 \mathrm{MW}$.

Of the 26 outages that cause between a $10 \mathrm{MW}$ and $50 \mathrm{MW}$ change in margin (mean change of $22 \mathrm{MW}$ ), the mean absolute errors of the linear and quadratic estimates are $14 \mathrm{MW}$ and 11 . MW respectively. The median absolute errors of the linear and quadratic estimates for these outages are $12 \mathrm{MW}$ and $8 \mathrm{MW}$ respectively.

The 25 worst outages resulting in at least a $50 \mathrm{MW}$ change in the margin are shown in Table 2. Estimates of a negative loading margin indicate that the formulas predict that no post-contingency solution exists for the base case loading at which the outage occurs. Both the linear and quadratic estimates select 20 of the 25 most severe outages and 10 of the worst 12 . Fifteen of the 25 most severe outages involve lines terminating at a transformer bus. Four of the 25 worst cases can be attributed to the effects of changing generator VAR limits (highlighted with slanted typeface). Of these four cases, only one can be somewhat anticipated from the quadratic estimates. With the exception of these four cases, both the quadratic and linear estimates perform well in ranking and grouping the outages.

\subsection{Multiple contingencies of 118 bus system}

Estimates of the effects of multiple contingencies are easily obtained. Linear estimates of multiple contingencies are simply sums of the linear estimates of the single contingencies making up the multiple contingency. Quadratic estimates of multiple contingencies are the sum of the quadratic estimates of the single contingencies together with cross terms accounting for interaction between contingencies.

The quadratic estimate is tested on the 118 bus system with 21 double line outages composed of combinations of 7 single line outages involving 8 buses. (The mean change in the loading margin of these 7 single line outages is $30 \mathrm{MW}$, and the mean absolute error in the quadratic estimates for these 7 single outages is $13 \mathrm{MW}$.) The mean change in the loading margin for the 21 double outages is $63 \mathrm{MW}$. The mean absolute error in the quadratic estimate is $32 \mathrm{MW}$. If the cross term is neglected, and the estimate found by simply summing the single line quadratic estimates, the mean absolute error is unchanged. For these contingencies, including the cross term of the quadratic estimate has a negligible effect on the accuracy of the estimates.

11 of the 21 double line outages cause a greater than $50 \mathrm{MW}$ reduction in the loading margin. When the 21 double line outages are considered along with the single line outages, the quadratic estimate selects 10 of the top 12 and 21 of the top 25 most severe contingencies.
Table 2: Estimated loading margins for the 25 worst outages of the 118 bus system.

nominal loading margin $=1766 \mathrm{MW}$

\begin{tabular}{|c|c|c|c|c|c|}
\hline \multirow{2}{*}{\multicolumn{2}{|c|}{$\begin{array}{l}\text { Line } \\
\text { Outage }\end{array}$}} & Linear & \multicolumn{3}{|l|}{ Quadratic } \\
\hline & & Estimate & Estimate & Exact $^{1}$ & Exact $^{2}$ \\
\hline & No. & MW (rank) & MW (rank) & MW (rank) & MW(rank) \\
\hline & 10 & -376 & -1635 & fatal & fatal \\
\hline 8 & 9 & -272 & -1531 & fatal & fatal \\
\hline 26 & 30 & 1502 & 1394 & 1318 & $1029 \quad(1)$ \\
\hline 4 & 5 & 1641 (2) & 1545 (2) & 1246 (1) & 1224 (2) \\
\hline 100 & 103 & $1766(132)$ & $1766(141)$ & $1766(139)$ & $1356 \quad(3)$ \\
\hline 25 & 27 & $1646(3)^{\prime}$ & 1571 & $1477 \quad(3)$ & $1468 \quad(4)$ \\
\hline 23 & 25 & 1691 & 1636 & 1493 (4) & 1488 (5) \\
\hline 69 & 75 & $1764(50)$ & $1762(48)$ & $1768(163)$ & $1582(6)$ \\
\hline 38 & 65 & 1664 & 1626 & $1603(5)$ & $1591(7)$ \\
\hline 22 & 23 & 1734 (11) & $1710(10)$ & $1638 \quad(7)$ & 1620 (8) \\
\hline 17 & 113 & $1702(6)$ & 1669 & 1633 & $1633(9)$ \\
\hline 3 & 5 & 1731 (8) & 1709 (8) & 1664 (8) & $1664(10)$ \\
\hline 11 & 13 & $1757(25)$ & $1750 \quad(19)$ & 1684 (13) & $1664(11)$ \\
\hline 8 & 30 & 1739 & 1751 (23) & $1668 \quad(9)$ & $1667(12)$ \\
\hline 88 & 89 & $1766(113)$ & $1766(121)$ & $1766(119)$ & $1669(13)$ \\
\hline 17 & 18 & $1742(14)$ & $1724(12)$ & $1670(10)$ & $1670(14)$ \\
\hline 64 & 65 & $1762(39)$ & 1758 & $1740(26)$ & $1671(15)$ \\
\hline 15 & 17 & $1731(10)$ & $1710 \quad(9)$ & $1676(11)$ & $1676(16)$ \\
\hline 30 & 38 & 1740 & 1742 (17) & $1683(12)$ & $1683(17)$ \\
\hline 21 & 22 & 1753 (19) & 1742 (16) & 1689 (16) & $1684(18)$ \\
\hline 4 & 11 & 1716 (7) & $1697 \quad(7)$ & 1686 (14) & $1686(19)$ \\
\hline 23 & 32 & 1731 (9) & 1712 (11) & 1688 (15) & $1687(20)$ \\
\hline 5 & 6 & 1745 (15) & 1732 (13) & 1704 (17) & $1704(21)$ \\
\hline 1 & 3 & 1760 & 1754 (27) & 1707 (18) & $1707(22)$ \\
\hline 2 & 12 & 1757 (24) & 1750 (18) & 1711 (19) & $1711(23)$ \\
\hline
\end{tabular}

1. VAR limited generators same as those at nominal nose. 2. VAR limited generators can differ from those at nominal nose.

\section{Discussion}

The 1390 bus results show that the sensitivity formulas are practical for ranking the severity of line outages in large power systems. In particular, the linear estimate takes very little time. Once the nominal point of collapse is found and a left eigenvector is obtained, 2000 single contingencies could be screened in less time than it takes to solve an average loadflow. The quadratic estimate could then be used to refine the estimates for those cases with the largest linear estimates. The computation time required for the quadratic estimate is approximately equal to the computation time required for an average loadflow.

The linear estimate identifies most severe contingencies. The quadratic term can improve the linear estimate, but only occasionally identifies a severe contingency missed by the linear estimate. Significant second order effects seem to be correlated to the outages of lines terminating at transformers, tie lines and their neighbors. Interarea flows are particularly sensitive to outages of tie lines and the flows from the high voltage 
networks to the low voltage networks are particularly sensitive to outages of lines terminating at transformers. These outages cause large power disturbances and would also be of concern for security issues other than voltage collapse.

The test results with both systems suggest that estimates whose quadratic term is small relative to the linear term are often closer approximations of the actual post-contingency loading margin than those for which the quadratic term is large compared to the linear term. The estimates almost always underestimate the severity of outages. The 118 bus results demonstrate that in some cases changes in VAR limits in force at the nose are significant.

This paper focuses on extracting the maximum information from a single nose curve. However, it might be desirable to establish more than one nominal collapse by running continuations from the base case for more than one pattern of loading ${ }^{1}$ or different assumptions concerning system operation. For example, since it may be possible to temporarily violate interarea agreements in emergencies, one might want to compute nominal noses both with and without area interchange enforced. This way, contingencies that cause problems under one set of assumptions but not others could be identified. Also, the nominal nose might be computed for different generator dispatches or load models. The parameter sensitivity methods in [4] can be used to select scenarios for which the loading margin is likely to vary significantly.

Generator outages could also be quickly ranked by computing the sensitivity of the loading margin to changes in generator power and dispatch. The results of [4] suggest that this method of ranking generator outages would work well for modest changes in power. Testing this method for larger changes in power is warranted.

\section{Computations}

For the computations of this paper, it is sufficient [26] to model the power system with static equations

$$
0=F(x, \lambda, p)
$$

where $x$ is the vector of equilibrium states, $\lambda$ a vector of load parameters, and $p$ a vector of parameters such as line admittances. $F$ should include area interchange, generator dispatch, and any other static controls. If a differential-algebraic or differential equation model of the power system is available then $F$ can be chosen as the right hand side of those equations.

For each single non-radial line outage the parameter vector $p$ is a vector with only three components - one each for conductance, susceptance, and shunt capacitance of the outaged line.

The first step in the computation is to obtain the projected direction of load increase from the short term

${ }^{1}$ The 118 bus results show a challenging case with high loading in several areas in which the ranking is imperfect partly because a few contingencies cause generator limits to change so that the voltage collapse area differs significantly from that of the base case. One approach to solve this difficulty monitors the effect of contingencies on several nose curves corresponding to loading predetermined voltage collapse areas. load forecast. For $\lambda_{0}$ the current vector of load parameters and $\lambda_{1}$ the forecasted short term load, the vector

$$
\hat{k}=\frac{\lambda_{1}-\lambda_{0}}{\left|\lambda_{1}-\lambda_{0}\right|}
$$

defines a unit vector in the direction of load increase.

The second step is to compute the nominal nose by a continuation method. During the continuation, the system equations change as limits such as reactive power limits apply.

The third step is to evaluate quantities at the nose and then, for each contingency, to evaluate the sensitivity formulas. For the rest of this section, (1) are the power system equations that apply at the nose. In particular, (1) accounts for the power system limits in force at the nose. The linear and quadratic formulas are derived for general parameter changes in [4].

\subsection{Linear estimate formula}

The linear estimate requires that the following quantities be computed at the nose:

- $w$, the left eigenvector corresponding to the zero eigenvalue of the system Jacobian $F_{x}$ ( $F_{x}$ evaluated at the nose is singular). It is practical and time saving to compute $w$ simultaneously with locating the nose.

- $F_{\lambda}$, the derivative of $F$ with respect to load parameters. For constant power loads $F_{\lambda}$ is a diagonal matrix with ones in the rows corresponding to load buses.

- $F_{p}$, the derivative of $F$ with respect to the line parameters evaluated at the nominal nose point. For a single line outage in any size system $F_{p}$ has three columns and only four (five for outage of a tie line) nonzero rows in the rows corresponding to the power balance equations at the buses connected by the line.

Let $\Delta p$ be the negative of the admittance vector for the line(s) to be outaged. Then the linear estimate for the change in margin [4] is:

$$
\Delta L=L_{p} \Delta p=\frac{w F_{p} \Delta p}{-w F_{\lambda} \hat{k}}
$$

The denominator of (3) is a scaling factor that is the same for all contingencies. The numerator of $(3)$ contains the vector $F_{p} \Delta p$ which, since $p$ appears linearly in $F$, is just the terms in $F$ that contain $p$. For $F$ representing real and reactive power balance, $F_{p} \Delta p$ is the vector of the pre-contingency real and reactive power injections on the outaged line. The linear formula is simply the power injections from the outaged lines scaled by the normalized left eigenvector $\tilde{w}=-w / w F_{\lambda} \hat{k}$ :

$$
\Delta L=\tilde{w}^{P_{i}} P_{i}+\tilde{w}^{Q_{i}} Q_{i}+\tilde{w}^{P_{j}} P_{j}+\tilde{w}^{Q_{j}} Q_{j}
$$

where $P$ and $Q$ are the pre-contingency real and reactive power injections to the outaged line, $i$ and $j$ indicate the buses connected by the outaged line, and $\tilde{w}^{P_{i}}$ represents the scaled left eigenvector component corresponding to real power balance at bus $i$. Formula (4) 
implies that lines with small flows are guaranteed to have small linear estirnates.

Radial line outages that isolate a portion of the network are a special case in which the power balance equations of the isolated bus should be deleted from $F$.

\subsection{Quadratic estimate formula}

The quadratic estimate additionally requires the following quantities evaluated at the nose:

- $v$, the right eigenvector corresponding to the zero eigenvalue of $F_{x}$.

- $w F_{x x}$, the matrix formed by product of $w$ with the Hessian tensor $F_{x x} . F_{x x}$ does not need to be found independently since: $w F_{x x}$ can be obtained as a byproduct of simultarieously solving for the exact nose point and $w$ with a direct method.

- $F_{x p}$, the derivative of the Jacobian with respect to the line parameters. For a typical single line outage $F_{x p}$ has at most 34 nonziero elements (16 each for the matrices corresponding to conductance and susceptance and 2 for the matrix corresponding to shunt capacitance). Since the line parameters appear linearly in the equilibrium equations, the nonzero elements are simple expressions of the voltages and angles at the buses connected by the outaged line.

- $X_{p}$, the sensitivity of the nose equilibrium with respect to $p$. ( $X$ is the position of the nose equilibrium; it varies with line parameters $p$.)

The quadratic estimate of the change in loading margin $[4]$ is

$$
\Delta L=L_{p} \Delta p+\frac{1}{2} \Delta p^{T} L_{p p} \Delta p
$$

where

$$
L_{p p}=\frac{X_{p}^{\prime} w F_{x x} X_{p}+2 w F_{x p} X_{p}}{-w F_{\lambda} \hat{k}}
$$

and $X_{p}$ is found by solving a sparse linear system with multiple right hand side:

$$
\left(\begin{array}{c}
F_{x} \\
w F_{x x} v
\end{array}\right) X_{p}=\left(\begin{array}{c}
-F_{p}-F_{\lambda} \hat{k} L_{p} \\
-w F_{x p} v
\end{array}\right)
$$

( $w F_{x x} v$ is a row vector). Note that the linear estimate for the resulting change in the nose equilibrium is

$$
\Delta X=X_{p} \Delta p
$$

and may be used to identify those contingencies likely to violate additional generator VAR or voltage limits prior to voltage collapse.(Generator reactive power outputs are components of $X$.)

When formulas (5) and (6) are applied to a double contingency, the number of parameters in $p$ and the dimensions of the vectors and matrices $L_{p}, L_{p p}$ double. The quadratic estimate for a double contingency takes into account interaction between the two contingencies and is different from the sum of the quadratic estimates for the corresponding two single contingencies.

\subsection{Computational efficiency}

This section analyzes the computational expense of the contingency analysis. For comparison, each step of the computations will be likened to one loadflow solution or one iteration of a loadflow solution. Generally, any technique used to speed the process of computing a loadflow solution or loadflow iteration could also be used to speed up the computation of the estimates. The slowest step in the procedure is the initial continuation to find the nominal nose. This continuation is only performed once, and any number of linear and quadratic estimates can be subsequently computed. The computational effort required for this continuation depends strongly on the system, the loading margin, the continuation algorithm, the desired accuracy, and assumptions about limits, and can range from several loadflow solutions to hundreds of loadflow solutions.

Quantities to be computed at the nominal nose only once are the left eigenvector $w$ and the scaling factor $w F_{\lambda} \hat{k}$ (the quadratic estimate also requires the right eigenvector $v$ ). The cost of computing each eigenvector is roughly equivalent to one loadflow iteration. The cost of computing $w F_{\lambda} \hat{k}$ is negligible.

The only computation required for each linear contingency estimate is finding the numerator of (3), and this just requires evaluating $F_{p} \Delta p$ at the nose and multiplying it by $w$. $F_{p} \Delta p$ could be obtained directly from the nominal loadflow computation (the pre-contingency flows on the outaged line), but computing it by sparse multiplication of $F_{p}$ and $\Delta p$ is also very fast. The computational effort to obtain $w F_{p} \Delta p$ for one contingency is less than 10 flops if $F_{p} \Delta p$ is found directly from the loadflow solution and at most 25 flops if $F_{p} \Delta p$ needs to be recomputed. This expense is independent of system size since $F_{p}$ always has only four or five nonzero rows. For an $N$ bus system with $2 N$ lines, obtaining all the linear estimates requires less than $20 \mathrm{~N}$ flops - typically less than one iteration of the loadflow for any system with more than just 20 buses. On a practical system, once a nominal nose and the left eigenvector have been found, linear estimates for all the line outages can be obtained more quickly than one loadflow solution.

The quadratic estimate requires the one time computation of $v, w F_{x x}$, and the factoring of the matrix $\left(\begin{array}{c}F_{x} \\ w F_{x x} v\end{array}\right)$ of (7). The quantities $v$ and $w F_{x x}$ are obtained from solution of the nominal nose at negligible cost. The matrix factoring costs less than one load flow iteration and need only be a partial refactoring since $F_{x}$ is already factored during solution of the nominal nose.

The dominant step in the evaluation of each quadratic estimate is obtaining $X_{p}$, which is found by solving the sparse linear system (7). The cost of setting up (7) for each contingency is just several sparse multiplications, less than $N$ flops. Solving for $X_{p}$ is roughly equivalent to three loadflow iterations. The remaining multiplications to establish the quadratic estimate require less than $N$ flops, so that each quadratic estimate is roughly as expensive as several iterations of the loadflow. The additional cost to compute any double contin- 
gency is negligible. The cost to compute one quadratic estimate for any single or double contingency is about equal to the cost of obtaining one loadflow solution.

\section{Conclusion}

This paper demonstrates that effective contingency analysis for voltage collapse can be done by computing the loading margin sensitivities for a nominal nose curve. This approach can take into account some of the effects of reactive power limits and easily handles multiple contingencies. The results show that the linear estimates are extremely fast and provide acceptable contingency ranking. For example, after the nose curve and a left eigenvector are computed, the linear estimates of all single line outages for a practical system are computed in less time than is typically required for one load flow solution. The quadratic estimates refine the linear estimates and are more costly but are still faster than previous methods. Similar sensitivity calculations [4] which exploit the same nose curve can be used to quickly select corrective actions to improve the loading margin to voltage collapse if the contingency analysis indicates a need for this.

\section{Acknowledgments}

We thank Claudio Cañizares for generous advice about computations. Funding in part from EPRI under contract WO8050-03 and from NSF PYI grant ECS9157192 is gratefully acknowledged.

\section{REFERENCES}

[1] Voltage stability of power systems: concepts, analytical tools, and industry experience, IEEE Special Publication 90TH0358-2-PWR, 1990.

[2] I. Dobson, H.-D. Chiang, Towards a theory of voltage collapse in electric power systems, Systems and Control Letters, vol. 13, 1989, pp. 253-262.

[3] L.H. Fink, ed., Proceedings: Bulk power system voltage phenomena III, voltage stability, security \& control, ECC/NSF workshop, Davos, Switzerland, August 1994.

[4] S.Greene, I.Dobson, F.L.Alvarado, Sensitivity of the loading margin to voltage collapse with respect to arbitrary parameters, IEEE Trans. Power Systems, vol.12, no.1, Feb. 1997, pp. 262-272.

[5] K. Iba et al., Calculation of critical loading condition with nose curve using homotopy continuation method, IEEE Trans. Power Systems, vol. 6, May 1991, pp. 584-593.

[6] V.Ajjarapu, C.Christy, The continuation power flow: a tool for steady state voltage stability analysis, IEEE Trans. Power Systems, vol.7, no.1, Feb.1992, pp.416-423.

[7] C.A. Cañizares, F.L. Alvarado, Point of collapse and continuation methods for large $\mathrm{AC} / \mathrm{DC}$ systems, IEEE Trans. Power Systems, vol.7, no.1, Feb.1993, pp.1-8.

[8] C. Rajagopalan, S. Hao, D. Shirmohammadi, M.K. Celik, Voltage collapse operating margin analysis using sensitivity techniques, Athens Power Tech,Athens,Greece,pp.332336,Sept.1993.

[9] H.-D. Chiang, A. Flueck, K.S. Shah, N. Balu, CPFLOW: A practical tool for tracing power system steady-state stationary behavior due to load and generation variations, IEEE Trans. Power Systems, vol.10, no.2, May 1995,pp.623-634.

[10] T.Wu,R.Fischl, An algorithm for detecting the contingencies which limit the inter-area megawatt transfer, North American Power Symp, Washington DC, pp.222-227,Oct.1993.

[11] F.L. Alvarado, Optimal planning of power systems, $\mathrm{PhD}$ thesis, University of Michigan, 1972.

[12] J. Peshcon, D. S. Percy, W. F. Tinney, O.J. Tveit, Sensitivity in power systems, IEEE Trans. Power Apparatus and Systems, vol PAS-87, no. 8, August 1968, pp. 1687-1696.
[13] M. Olofsson, Optimal operation of the Swedish railway electrical system, $\mathrm{PhD}$ thesis, Royal Institute of Technology, Stockholm Sweden, TRITA-EES-9601, ISSN 11001607, February 1996.

[14] H. Mori, H. Tanaka, J. Kanno, A preconditioned fast decoupled power flow method for contingency screening, IEEE Trans. Power Systems, vol.11, no.1, Feb.1996,pp.357-363.

[15] O. Penangsang, Fast linear screening approach for contingency selection of line outages based on voltage quality, PhD Thesis, University of Wisconsin-Madison, May 1983.

[16] F. Albuyeh, A. Bose, B. Heath, Reactive power considerations in automatic contingency selection, IEEE Trans. Power Apparatus and Systems, vol PAS-101, no. 1, January 1982, pp. 107-111.

[17] K.Nara, K. Tanaka, R.R. Shoults, M.S. Chen, P. Van Olinda, On-line contingency selection algorithm for voltage security analysis, IEEE Trans. Power Apparatus and Systems, vol PAS-104, no. 4, April 1985, pp. 847-856.

[18] G.C.Ejebe, H.P.Van Meeteren, B.F.Wollenberg, Fast contingency screening and evaluation for voltage security analysis, IEEE Trans. Power Systems, vol.3, no.4, Nov. 1988, pp. 1582-1590.

[19] N. Flatab $\varnothing$, O.B. Fosso, R. Ognedal, T. Carlsen, K.R. Heggland, A method for calculation of margins to voltage instability applied on the Norwegian system for maintaining required security level, IEEE Trans. Power Systems, vol. 8, no. 2, May 1993, pp. 920-928.

[20] O.B.Fosso,N.Flatab $\phi, B . H a k a v i k, A . T . H o l e n$, Comparison of methods for calculation of margins to voltage instability,Athens Power Tech, Athens,Greece,pp.216-221,Sept.1993

[21] N.D.Reppen,R.R.Austria,J.A.Uhrin,M.C.Patel,A.Galatic, Performance of methods for ranking and evaluation of voltage collapse contingencies applied to a large-scale network, Athens Power Tech, Athens, Greece,pp.337-343,Sept.1993.

[22] G.C. Ejebe, G.D. Irisarri, S. Mokhtari, O. Obadina, P. Ristanovic, J. Tong, Methods for contingency screening and ranking for voltage stability analysis of power systems, IEEE Trans. Power Systems, vol.11, no.1, Feb.1996, pp.350-356.

[23] L. Wehenkel, Contingency severity assessment for voltage. security using non-parametric regression techniques, IEEE Trans. Power Systems, vol.11, no.1, Feb.1996, pp.101-111.

[24] H-D Chiang, C-S Wang, A.J. Flueck, Look-ahead voltage and load margin contingency selection functions for largescale power systems, IEEE Trans. on Power Systems, vol. 12, no. 1, Feb.1997, pp.173-180.

[25] Z.C. Zeng, F.D. Galiana, B.T. Ooi, N. Yorino, A simplified approach to estimate maximum loading conditions in the load flow problem, IEEE Trans. Power Systems, vol. 8, no. 2, May 1993, pp. 646-653.

[26] I. Dobson, The irrelevance of load dynamics for the loading margin to voltage collapse and its sensitivities, in [3].

27] Data available via ftp at wahoo.ee.washington.edu

28] PFLOW: Software available via anonymous $\mathrm{ftp}$ at iliniza uwaterloo.ca in subdirectory pub/pflow

[29] F.L. Alvarado, Manipulation and visualization of sparse matrices, ORSA (Operations Research Society of America) Journal on Computing, 2(2):186-207, Spring 1990.

[30] Sparse Matrix Manipulation System: Users manual and software are available via anonymous $\mathrm{ftp}$ at eceserv0.ece. wisc.edu in subdirectory pub/smms93

Scott Greene received the BS in Mechanical Engineering from Cornell University in 1984, and the MS in Electrical Engineering from the University of Wisconsin-Madison in 1993, where he is a candidate for the PhD in Electrical Engineering. He has worked as an electrical and mechanical systems engineer at Strand Associates of Madison, Wisconsin and the General Electric Company. He is a registered professional engineer in Wisconsin.

Ian Dobson received the $\mathrm{PhD}$ in electrical engineering from Cornell University in 1989 and is now an associate professor of electrical engineering at the University of Wisconsin-Madison. His interests are applications of bifurcations and nonlinear dynamics, electric power system stability and utility power electronics.

Fernando Alvarado obtained the Ph.D. from the University of Michigan in 1972. He is currently a Professor at the University of Wisconsin in Madison in the Department of Electrical and Computer Engineering. His main areas of interest are computer applications to power systems and large scale problems. 


\section{Discussion}

Anjan Bose and Hang Liu (Washington State University, Pullman, WA): The authors are congratulated for presenting a fast contingency ranking method for voltage collapse analysis. The proposed method is based on the sensitivity analysis of load margin with respect to the contingency line parameters. The time gain is achieved because only one nominal nose curve is calculated and the sensitivity computations are also fast. The authors' comments on the following issues would be appreciated:

1. Both linear and quadratic estimation methods proposed use the linearized system information (the sensitivity $\mathrm{L}_{\mathrm{p}}$ ). In general, this kind of linear analysis is suited for small system disturbances (i.e., small parameter changes). However, system contingencies are considered to be large disturbances, especially for a stressed line. This may explain the errors, which are sometimes quite large, in the load margin calculations in Table I. The proposed method may have some inherent limitations in its application to such cases.

2. Also, these results in Table I seem to consistently provide an optimistic load margin when compared to the exact load margin. However, a relatively conservative method is preferred in security analysis. If indeed this error is always in one direction and some error bound for linearization can be estimated, then a compensation factor can be developed to ensure conservatism in the answer. Otherwise, the results may be a bit too erratic on which to base corrective action.

3. As the authors point out, the calculation of the load margin assumes that the contingency nose curve corresponds to the collapse of the voltage(s) of the same bus(es) as in the base case. This is a rather restrictive assumption, especially for a large system or a system that is stressed in more than one area at the same time. Thus one nose curve for the base case may not be enough to represent and analyze all contingencies for a very large stressed system. However, a procedure to choose more than one base case nose curve, presumably obtained by using different loading parameters, is not obvious.

Scott Greene, Ian Dobson, Fernando Alvarado: We thank Anjan Bose and Hang Liu for their pertinent comments.

1. We emphasize that this paper describes a fast method of ranking contingencies for voltage collapse rather than a method of accurately determining the post-contingency loading margins to voltage collapse. (The most accurate type of method for computing the post-contingency loading margins to voltage collapse is the continuation used in the paper to test the ranking estimates.) We agree that there are errors when using the sensitivity formulas to estimate the size of the change in loading margin due to contingencies. The errors in the change in the loading margin are in part due to the approximation of a large deviation by the first (or first two) terms of a Taylor series as suggested by the discussers. However, the paper describes a second notable source of error: if the reactive power limits in force at the nose change, this causes the equations at the nose to change. Even if the error due to a large deviation in a fixed set of nonlinear equations were eliminated, the second error due to changes in the equations would remain. Indeed, the 118 bus results show that computing the post-contingency loading margins by a continuation method that assumes the limits remain fixed (this is equivalent to a perfect large deviation estimate) still misranks the same contingencies as the linear or quadratic estimates. The presence of the second error limits the benefits of minimizing the large deviation error.

It would still be desirable to minimize the large deviation error if this could be done with a sufficiently fast method. One different way to apply the margin sensitivities is to evaluate them with respect to changes in the impedance matrix resulting from the line outage. The exact impedance matrix changes are computed with a large deviation method using a sparse matrix version of a rank one update [A1,A2]. Preliminary tests show that this large deviation method does provide bigger estimates and often overestimates the changes in loading margin. However, the large deviation estimates appear to be no better at ranking than the linear estimate. The large deviation method is faster than the quadratic estimate but slower than the linear estimate of this paper. Our preliminary tests are not as encouraging as we had hoped but this idea or other variants might be worth investigating.

2. The discussers indicate that the estimates consistently underestimate the change in loading margin. For purposes of ranking, consistency is an asset. While the methods occasionally give a false negative, they do not tend to give false positives. The contingencies ranked highly are serious contingencies. The discussers suggestion of a compensation factor is sensible and might be effective on specific systems.

Contingency ranking methods trade off speed and accuracy. The linear estimate is an extremely fast method of obtaining useful ranking estimates but is of limited accuracy in estimating the change in margin. While there certainly are opportunities for slower and more accurate methods to be developed, these would be most efficiently applied after a fast initial screening.

One approach available now for developing corrective actions would be to perform an initial screening with our linear estimate and then recompute nose curves for the worst contingencies to obtain the actual change in margin. The effects of corrective actions can then be very quickly estimated using the formulas of reference 4 evaluated at the new noses.

3. This point relates to the footnote halfway through the paper. The 118 bus example was chosen to be a challenging case for our methods in this respect. More typical 
cases might be expected to yield improved results. We agree that a sensible way to treat cases in which different areas collapse for different contingencies is to compute several nominal nose curves. If the vulnerable areas and the corresponding loadings leading to their collapse are not already known, one (untested) suggestion to compute them is as follows: If the base case loading is not a stressed system then obtain a new, stressed base case using the previous loading direction. Running the worst case loading algorithm [A3] from the new base case for several initial loading directions should yield areas close to collapse and their corresponding loading directions. Plausible initial loading directions can be found by modifying the previous loading direction by not increasing the load at buses corresponding to previously found areas.

We remark that our approach of measuring a margin accurately by continuation and then approximating the change in margin by sensitivity formulas evaluated at the nose contrasts with the usual approach to contingency ranking of estimating at the operating point changes due to contingencies. The new approach is necessary for voltage collapse because of the importance for voltage collapse of nonlinearity and generator reactive power limits. Both these factors are taken into account when computing the nominal loading margin by continuation. Although our paper is one of the first attempts to solve the ranking problem for voltage collapse, we suspect that the linear sensitivity method will be a strong contender for the fastest ranking method of good enough accuracy. At the same time, despite the challenges inherent in voltage collapse, there is scope for ingenuity in improving accuracy, probably at the expense of speed.

Similar sensitivity methods apply to available transfer capabilities. The recent increased focus on available transfer capabilities makes it worthwhile to improve the handling of nonlinearity for power system operating limits other than voltage collapse. One approach measures margins to these other operating limits by continuation and applies margin sensitivity formulas at the operating limits [A4].

\section{References}

[A1] O. Alsac, B. Stott, W.F. Tinney, Sparsity oriented compensation methods for modified network solutions, IEEE Trans. Power Apparatus and Systems, vol. PAS-102, May 1983, pp.1050-1060.

[A2] B. Stott, O. Alsac, F.L. Alvarado, Analytical and computational improvements in performance-index ranking algorithms for networks, International Journal of Electrical Energy and Power Systems, vol. 7, no. 3 , July 1985 , pp.154-160.

[A3] F.L. Alvarado, I. Dobson, Y. Hu, Computation of closest bifurcations in power systems, IEEE Trans. Power Systems, vol. 9, no. 2, May 1994, pp. 918-928.

[A4] S. Greene, I. Dobson, F.L. Alvarado, P.W. Sauer, Initial concepts for applying sensitivity to transfer capability, NSF Workshop on Available Transfer Capability, Urbana IL USA, June 1997. 